\title{
Comment on: An Expanded View of Complex Traits: From Polygenic to Omnigenic
}

\author{
Xin $\mathrm{He}^{1^{*}}$ \\ ${ }^{1}$ Department of Human Genetics, University of Chicago. \\ *Correspondence: Xin He, Email: xinhe@uchicago.edu.
}

The recent paper by Pritchard and colleagues proposed an "omnigenic" model to explain genetic architecture of complex human traits. The model states that a trait may be directly affected by a small set of "core genes", but many other genes may perturb the activity of the core genes because of the inter-connectivity of biological networks. I believe this is an extremely important paper. The proposed model could serve as a conceptual framework that unifies many observations in genetics. For example, it explains why genes found in rare variant studies often have clear disease connection while GWAS do not (only large-effect genes can be found in rare variant studies because of lower power); why variants often have pleiotropic effects on multiple traits; and why polygenic adaptation is common.

While it is conceptually important, the paper presents relatively light evidence in direct support of the omnigenic model. One main argument is that biological networks are highly connected, so it is plausible and perhaps common for one gene to affect the function of another seemingly-unrelated gene. I found this argument somewhat speculative. There is a large amount of literature supporting the robustness of biological systems: that they are often insensitive to genetic and environmental perturbations ${ }^{[1]}$. The phenomenon of "canalization" is well known in the field of developmental biology. Biological systems have evolved many design features that make them robust, and as a result, perturbation in one node is not necessarily propagated to other nodes in the networks. For example, the activity of a protein is regulated at many levels such as transcription initiation, mRNA stability and translation, so the changes at one level can often be compensated by another to maintain the protein activity. Biological networks often have negative feedback loops and other features that stabilize the network output, often activities of key genes. It remains to be seen how the robustness of biological systems reconciles with the assumption underlying the omnigenic model.

There could also be alternative explanations of the body of evidence that prompted the authors' model. For example, acknowledging that there are a set of core genes of a disease, many other genes may affect the disease risk, not through acting on core genes, but by altering cellular states: how fast cells divide, how tolerant they are to stress, how quickly they respond to hormones, etc. These cellular states may be subject to many genetic influences, and if perturbed, may have non-negligible impact on disease risks. Such a model will also lead to polygenicity of traits (many ways of altering cellular states), 
widespread pleiotropy (the same cellular states may be involved in many diseases) and polygenic adaptation (consequence of polygenecity of cellular states).

I believe it is thus important to test the omnigenic model against alternative explanations such as the "cellular state" model above. One specific and important prediction of the omnigenic model is that many disease loci that act on peripheral genes are also trans-acting QTL on core genes. If we use gene expression as a measure of activity, the model predicts that many disease loci would converge on the same core genes as trans-eQTL. While there are few published studies linking trans-eQTL and diseases, the available papers do provide some support on this prediction. Westra et al. reported 21 complex traits where multiple unlinked disease SNPs affecting expression of the same downstream target genes in trans (Table 2 in the paper) ${ }^{[2]}$. The Sherlock method proposed by myself explicitly searches for genes where multiple eQTL (both cis and trans) are also disease-associated SNPs ${ }^{[3]}$. One top gene for Crohn's disease, for instance, has four transeQTL, each associated with a modest disease risk (EFS, see Table 2). The EFS gene seems to satisfy the definition of core genes: it has a clear immune function and has phenotype in mice resembling Crohn's disease. While Sherlock can be a useful tool to study the omnigenic model, it has several limitations, e.g. it does not take the effect size of a gene into account, missing an important aspect of the omnigenic model (core genes should have large effects). I believe an important research direction, both in method development and in empirical studies, is to exploit trans-acting QTL to search for core genes and to test the omnigenic model.

\section{REFERENCES}

1. Wagner A. Robustness and Evolvability in Living Systems. New Jersey: Princeton University Press; 2005.

2. Westra HJ, Peters MJ, Esko T, Yaghootkar $H$, Schurmann C, Kettunen J, et al. Systematic identification of trans eQTLs as putative drivers of known disease associations. Nat Genet. 2013; 45(10): 1238-1243.

3. He X, Fuller CK, Song $Y$, Meng Q, Zhang B, Yang $X$, Li H. Sherlock: detecting gene-disease associations by matching patterns of expression QTL and GWAS. Am J Hum Genet. 2013; 92(5): 667-680. 